Marcio Alazraqui ${ }^{1}$

Hugo Spinelli ${ }^{1}$

Marina Gabriela Zunino ${ }^{1}$

Edinilsa Ramos de Souza ${ }^{2}$

\section{Calidad de los sistemas de información de mortalidad por violencias en Argentina y Brasil - 1990-2010}

\author{
The quality of information systems on violence-related deaths \\ in Argentina and Brazil between 1990 and 2010
}

Instituto de Salud Colectiva, Universidad Nacional de Lanús. Av. 29 de Septiembre 3901, edificio

"Leonardo Werthein",

Remedios de Escalada. 1826 Lanús Provincia de Buenos Aires Argentina.

malazraqui@yahoo.com.br ${ }^{2}$ Escola Nacional de Saúde Pública, Fundação Oswaldo Cruz.
Abstract Violence-related deaths are a public health issue by virtue of the magnitude of their impact on society and on the health services. A study of the descriptive temporal trend was conducted using the official national information systems of violence-related mortality in Argentina and Brazil for the period from 1990 to 2010. Indicators were created to evaluate information quality by sex, age, and cause of death. The results demonstrate a temporal trend of improvement in the quality of the information systems. This trend is repeated especially when violencerelated deaths are analyzed, with a decrease in the number of records for violence-related death of undetermined intent; as well as a high percentage of firearm-related deaths of undetermined intent in Argentina. The analysis of the quality of information systems regarding violence-related deaths makes it possible to detect problems and orient actions in order to obtain better quality information and therefore permit improvement in the creation of preventive public policies.

Key words Violence, External causes, Information systems, Mortality statistics, Temporal trends.
Resumen Las muertes por violencias son un problema de salud pública por la magnitud de su impacto social y en los servicios de salud. Se realizó un estudio descriptivo de tendencia temporal a partir de los sistemas de información oficiales nacionales de mortalidad por violencias en Argentina y Brasil en el periodo 1990-2010. Se elaboraron indicadores de la calidad de la información según sexo, edad y causa de defunción. Los resultados muestran una tendencia temporal a la mejoría de calidad en los sistemas de información. Esta tendencia se repite cuando analizamos las muertes por violencias en particular, con una disminución de los registros por muertes por violencias de intencionalidad ignorada; y un porcentaje alto de muertes por armas de fuego de intencionalidad ignorada en Argentina. El análisis de la calidad de los sistemas de información de mortalidad por violencias permite detectar problemas y orientar acciones para obtener información de mayor calidad a fin de orientar politicas públicas preventivas.

Palabras clave Violencias, Causas externas, Sistemas de información, Estadisticas de mortalidad, Tendencia temporal 


\section{Introducción}

Las muertes por violencias son un problema de salud pública ${ }^{1}$ por la magnitud de su impacto social y en los servicios de salud ${ }^{2-8}$. La comprensión de este problema complejo debiera orientar políticas públicas para su enfrentamiento. Desde el sector salud una aproximación al problema es a través de las estadísticas de mortalidad.

En las décadas de 1960-70, varios países de América Latina, como Argentina y Brasil, implementaron sistemas de información de estadísticas vitales, dónde se incluyen las estadísticas de mortalidad que ya existían previamente. Los sistemas de información estadísticos de mortalidad de ambos países tienen una estructura y organización semejantes. El órgano rector es el Ministerio de Salud y tiene una cobertura universal amplia. El instrumento utilizado es semejante en ambos países, en Brasil es la "Declaração de Óbito" y en Argentina el "Informe Estadístico de Defunción”. Cuando se trata de una muerte por violencia interviene en ambos casos la figura del médico legista. Los registros civiles de los diferentes municipios obtienen la información de mortalidad y la remiten a niveles superiores, como las provincias o estados, para finalmente consolidarse a nivel nacional. Es decir, se trata en ambos casos de un sistema de información continuo descentralizado que envía información al nivel nacional.

Las estadísticas de mortalidad registran todas las muertes ocurridas en la población, incluidas las muertes por violencias, denominadas Causas Externas por la Clasificación Internacional de Enfermedades ${ }^{9-10}$. Esta denominación es utilizada ampliamente, aunque se trata de un término que ha suscitado críticas ${ }^{11-12}$. Las causas externas pueden dividirse según la intencionalidad del hecho en: suicidios, homicidios, accidentes y de intencionalidad ignorada. En esta última categoría se ignora si la intencionalidad de la muerte por violencia fue intencional (suicidio u homicidio) o accidental (accidentes). La importancia de la magnitud de las causas externas de intencionalidad ignorada radica en que se trata de alguno de los otros tres tipos descriptos, que por este motivo podría encontrarse sub-dimensionado al realizar el cálculo del indicador correspondiente, por ejemplo: puede ocultar homicidios ${ }^{13}$ y más aún cuando se trata de defunciones producidas por un arma de fuego ${ }^{14-16}$.

Argentina y Brasil tienen tasas de mortalidad por causas externas de diferente magnitud, en especial en las tasas de homicidios ${ }^{17}$. La tenden- cia temporal de los homicidios puede estar afectada por la calidad del sistema de información de mortalidad y en especial por la magnitud de defunciones por causas externas cuya intencionalidad se ignora. Por este motivo nos proponemos analizar la calidad de la información sobre muertes por violencias derivada del sistema de estadísticas de mortalidad. Se analizan también la calidad de las muertes por violencias cuya intencionalidad se ignora según sexo debido a la relevancia de la violencia doméstica ${ }^{18-20}$

La base conceptual común de las estadísticas de mortalidad (incluidas en las estadísticas vitales), implementada de forma extendida en el mundo, tiene como uno de sus objetivos la comparación de la estructura de mortalidad entre países a fin de entender sus diferencias y orientar políticas públicas que incrementen el nivel de salud de las poblaciones. Sin embargo existen pocos estudios comparativos en lo que respecta a la calidad de la información en muertes por violencias.

Esta investigación tiene como objetivo el análisis descriptivo de la tendencia temporal de la calidad de los registros estadísticos de mortalidad por violencias en Argentina y Brasil en el periodo 1990-2010.

\section{Metodología}

Se realizó un estudio epidemiológico descriptivo de tendencia temporal de la calidad de los registros estadísticos de mortalidad en Argentina y Brasil durante el período 1990-2010. Se focalizó el interés a partir del año 1990 a fin de describir la tendencia de la calidad de la información de mortalidad en los últimos años.

Las fuentes de información fueron los sistemas de información sobre mortalidad de los países involucrados, que forman parte de las estadísticas vitales y abarcan todas las muertes ocurridas. En Argentina los datos provienen de los Informes Estadísticos de Defunción del Sistema de Estadísticas Vitales de la Dirección de Estadística e Información en Salud (DEIS) del Ministerio de Salud de la Nación; en Brasil los datos fueron obtenidos del Sistema de Información de Mortalidad (SIM) del Departamento de Informática del Sistema Único de Salud (DATASUS). Se obtuvieron las bases de datos de mortalidad para su análisis, en formato electrónico en todos los casos.

Con la intención de verificar la calidad de los registros de mortalidad utilizados se realizó un análisis descriptivo de las variables sexo, edad y 
de agrupaciones seleccionadas de la causa básica de defunción. Los datos referentes al período de 1990 a 2010 fueron recolectados para cada país según sexo (masculino o femenino) y grupos etarios (0-9, 10-14, 15-19, 20-29, 30-39, 40-49, 5059 o 60 y más años). Se utilizó la Clasificación Internacional de Enfermedades (CIE) para codificar la causa básica de defunción ${ }^{9-10}$. Dado que a partir de 1997 la Organización Mundial de la Salud realizó modificaciones a la mencionada clasificación, se utilizaron las equivalencias entre la IX Revisión (CIE-IX) y la X Revisión (CIE-X) ${ }^{21-22}$. La codificación de la causa básica de defunción, cuando se trata de una muerte por violencias, tiene en cuenta la intencionalidad y las circunstancias asociadas ${ }^{9-10}$.

Los agrupamientos de causa básica de defunción seleccionados fueron los siguientes: a) causas mal definidas (códigos CIE-IX: 780-799, y CIE-X: R00-R99); b) causas externas (códigos CIE-IX: E960-E969, y CIE-X: X85-Y09, Y87.1); c) causas externas de intención no determinada (códigos CIE-IX: E980-E989, y CIE-X: Y10-Y34, Y87.2, Y89.9); d) causas externas de intención no determinada debidas a disparo de arma de fuego (códigos CIE-IX: E985 y CIE-X: Y22-Y24). El análisis de estos dos últimos grupos de causas nos permite una interpretación más adecuada de las tasas por homicidios (códigos CIE-IX: E960E969, y CIE X: X85-Y09 e Y87.1 $)^{9-10}$.

El análisis estadístico consistió en la elaboración de indicadores que den cuenta de la calidad de la información de mortalidad por todas las causas, y también específicamente cuando se trata de causas por violencias. Los indicadores seleccionados fueron los siguientes: a) porcentaje de registros de mortalidad sin información de sexo y/o edad del total de defunciones; b) porcentaje de registros de mortalidad con codificación inespecífica de la causa básica de defunción ("mal definidas") del total de defunciones; c) porcentaje de registros sin información sobre la intencionalidad del hecho violento del total de causas externas, según sexo; d) porcentaje de registros sin información sobre la intencionalidad del hecho violento que fueron debidos a armas de fuego del total de registros sin información sobre la intencionalidad del hecho violento, según sexo.

El análisis descriptivo de los datos de mortalidad fue presentado en tablas y figuras de tendencia temporal para las variables principales. Los programas utilizados en la investigación fueron: Microsoft Excel 2010 ${ }^{\circledR}$ y Microsoft Access $2010^{\circledR}$.
Se cumplieron normas éticas respetando las leyes de secreto estadístico y preservando la confidencialidad de las personas involucradas en las bases de datos de mortalidad (Argentina: Ley No 17.622 del 25/01/68; y Brasil: Ley No 5.534 del 14/ $11 / 68$ ). Se trata además de bases de datos a nivel individual que no tienen identificación de los involucrados. Por estos motivos no fue necesaria una aprobación específica de los comités de ética de la investigación en ambos países.

\section{Resultados}

Se analizaron las estadísticas de mortalidad de Argentina y Brasil durante el periodo 1990-2010 (21 años).

En primer lugar se presentan indicadores de la calidad de las estadísticas de mortalidad en general y luego se presentan indicadores de la calidad de las estadísticas de mortalidad específicos de las muertes por violencias.

La Figura 1 presenta el análisis descriptivo de la tendencia temporal del porcentaje de registros de mortalidad sin información de sexo y/o edad del total de defunciones. Observamos una tendencia decreciente a lo largo del periodo en estudio. Es de destacar que en cualquier año del periodo el porcentaje sin información de sexo o edad en los registros de defunción es menor al 2,7\% en Argentina y menor al 1,6\% en Brasil. Los valores de los dos países parten de niveles semejantes $y$, a pesar de algunas oscilaciones, al final del periodo tienen valores muy parecidos, alrededor del 0,4\%.

En la Figura 2 observamos el porcentaje de registros de mortalidad con codificación inespecífica de la causa básica de defunción ("mal definidas") en relación al total de defunciones por todas las causas. En Brasil la tendencia temporal descendiente es muy marcada; mientras en Argentina se observa un nivel semejante a lo largo del periodo (con un leve aumento a partir de 2002, con un posterior descenso a partir de 2008). Al final del periodo se observan niveles muy semejantes entre Argentina y Brasil. Esta tendencia indica que la calidad de la certificación de la causa básica de defunción ha mejorado durante el periodo de estudio de forma franca en Brasil; y se ha mantenido estable en Argentina.

A continuación presentaremos indicadores de la calidad de la información de los registros de mortalidad correspondientes a causas externas.

La Figura 3 muestra el análisis descriptivo de la tendencia temporal del porcentaje de muertes 


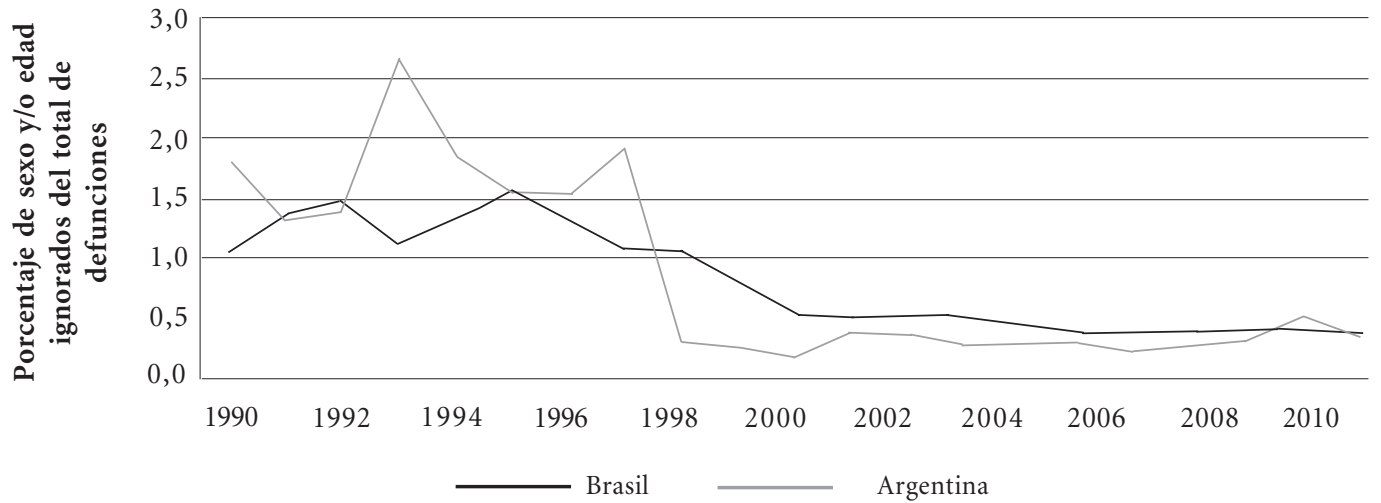

Figura 1. Tendencia temporal del porcentaje de registros estadísticos de mortalidad sin información de sexo y/ o edad del total de registros (todas las causas). Argentina y Brasil, 1990-2007.

Fuente: Elaboración propia a partir las bases de datos de mortalidad de DEIS-MSN (Argentina) y SIM-DATASUS (Brasil).

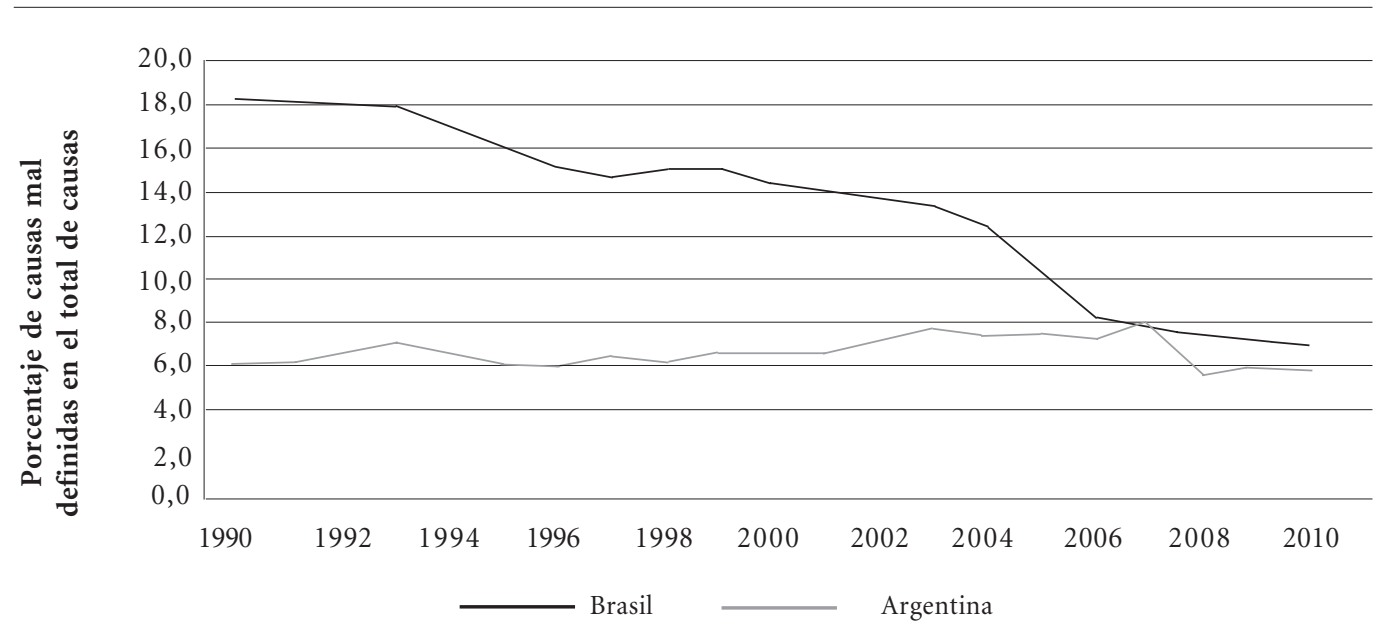

Figura 2. Tendencia temporal del porcentaje de registros estadísticos de mortalidad sin información de la causa básica de defunción del total de registros (todas las causas). Argentina y Brasil, 1990-2007.

Fuente: Elaboración propia a partir las bases de datos de mortalidad de DEIS-MSN (Argentina) y SIM-DATASUS (Brasil).

por violencias sin información sobre la intencionalidad del hecho (del total de causas externas) para los dos países. Argentina presenta valores relativos y oscilaciones en la tendencia temporal que son de mayor magnitud que en Brasil. En relación al comienzo del periodo los porcentajes tienden a mejorar levemente en Brasil; y a empeorar levemente en Argentina.

La Figura 4 muestra el análisis descriptivo de la tendencia temporal del porcentaje de muertes por violencias sin información sobre la intencio- nalidad del hecho (del total de causas externas) teniendo en cuenta el sexo (hombres y mujeres por separado). En Argentina observamos los mayores valores en 1995 y los menores en 2005, con un leve aumento al final del periodo (2010), y una tendencia temporal semejante en hombres y en mujeres (aunque los porcentajes y los valores absolutos son significativamente mayores en los hombres). En Brasil se presenta una leve tendencia a la disminución del porcentaje de violencias de intencionalidad ignorada en hombres y 


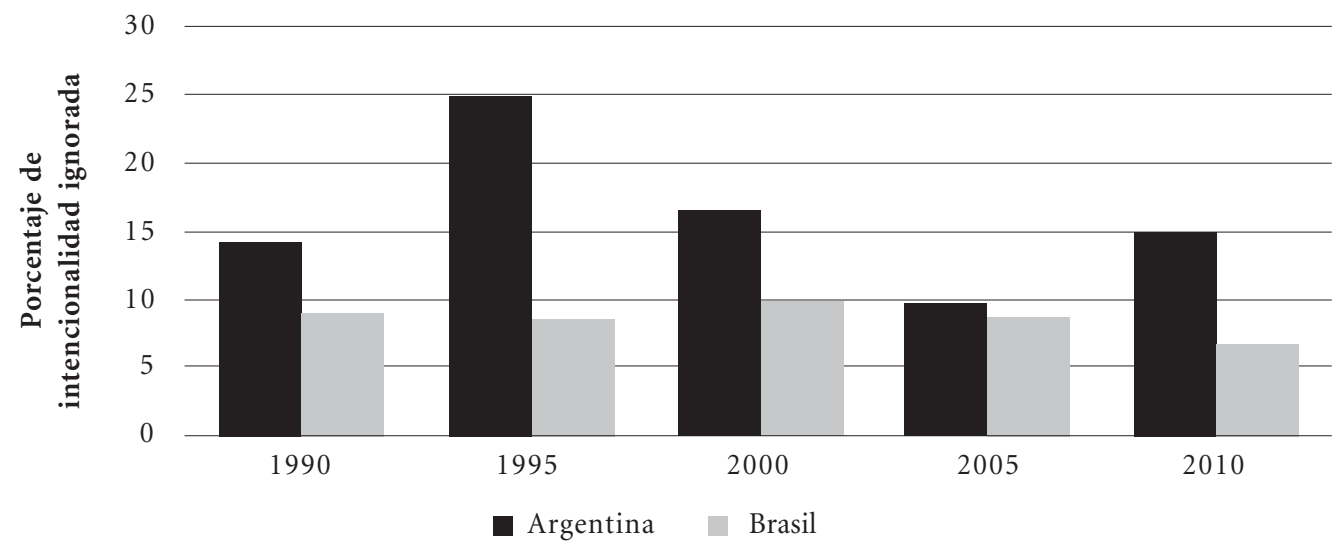

Figura 3. Tendencia temporal del porcentaje de registros estadísticos de mortalidad de defunciones por lesiones que se desconoce si fueron accidentales o intencionalmente infligidas en ambos sexos. Argentina y Brasil, 1990, 1995, 2000, 2005 у 2010.

Fuente: Elaboración propia a partir las bases de datos de mortalidad de DEIS-MSN (Argentina) y SIM-DATASUS (Brasil). Nota: Las defunciones por lesiones que se desconoce si fueron accidentales o intencionalmente infligidas corresponden a los códigos CIE-IX: E980-E989; y CIE-X: Y10-Y34, Y87.2, Y89.9.

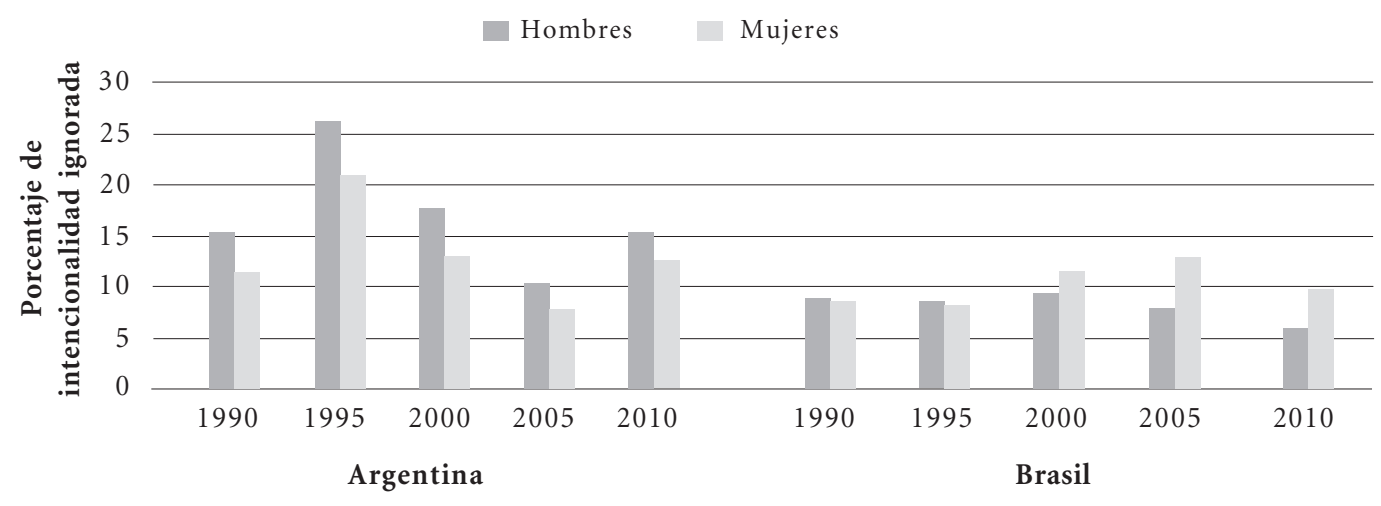

Figura 4. Tendencia temporal del porcentaje de registros estadísticos de mortalidad de defunciones por lesiones que se desconoce si fueron accidentales o intencionalmente infligidas en hombres y mujeres. Argentina y Brasil 1990, 1995, 2000, 2005 y 2010.

Fuente: Elaboración propia a partir las bases de datos de mortalidad de DEIS-MSN (Argentina) y SIM-DATASUS (Brasil). Notas: a) Las defunciones por lesiones que se desconoce si fueron accidentales o intencionalmente infligidas corresponden a los códigos CIE-IX: E980-E989; y CIE-X: Y10-Y34, Y87.2, Y89.9. b) Los porcentajes son calculados sobre el total de muertes por violencias para cada sexo.

mujeres al final del periodo; mientras observamos un aumento en mujeres hasta 2005. Es de tener en cuenta que este leve aumento en mujeres tiene una magnitud que no influye significativamente en la tendencia del total, y que disminuye al final del periodo en estudio. Los porcentajes presentados en Brasil son inferiores a Argentina, con la excepción de las mujeres en 2005.
La Figura 5 muestra la magnitud de las muertes por armas de fuego en las causas externas de intencionalidad ignorada que se analizan porque representan una parte importante de este grupo de causas. En Brasil los niveles son bastante menores que en Argentina en todo el periodo. Argentina presenta los mayores valores en 20022003. Es de destacar que los porcentajes y valores 


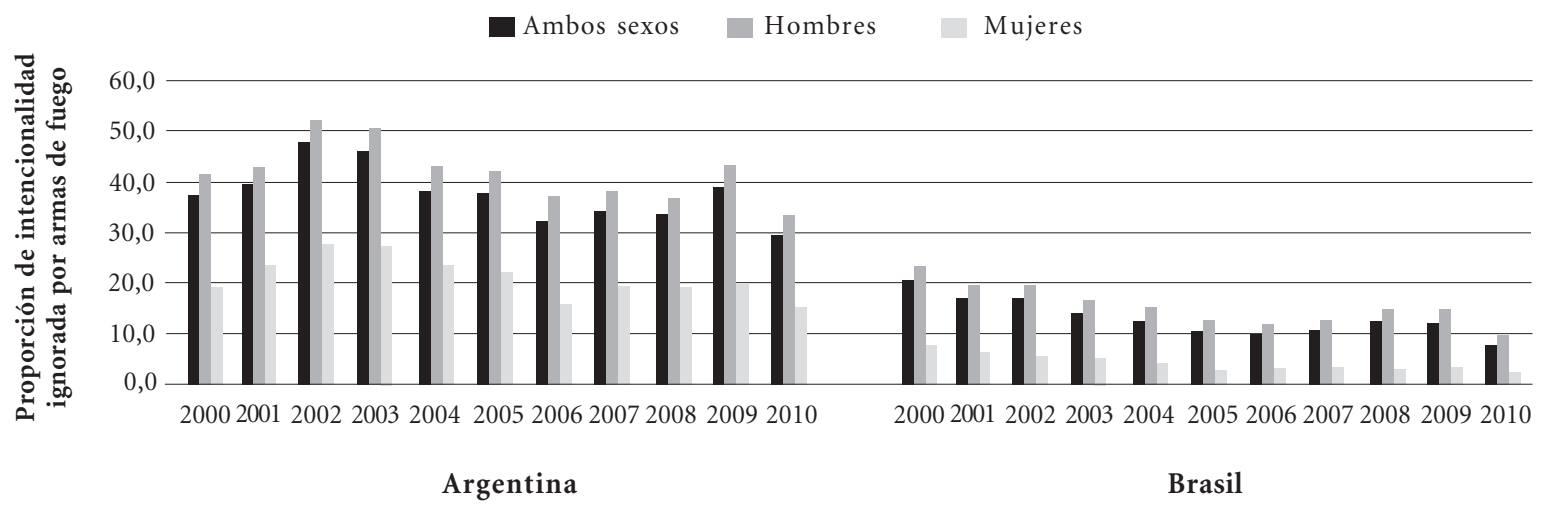

Gráfico 5. Tendencia temporal del porcentaje de registros de mortalidad debidos a armas de fuego del total de registros sin información sobre la intencionalidad del hecho violento, según sexo. Argentina y Brasil, 2000-2010.

Fuente: Elaboración propia a partir las bases de datos de mortalidad de DEIS-MSN (Argentina) y SIM-DATASUS (Brasil). Nota: Los porcentajes son calculados sobre el total de muertes por violencias de intencionalidad ignorada, para cada sexo.

absolutos son mayores en hombres en ambos países.

Se encuentra también que en los últimos años de la serie presentada las edades predominantes en las muertes por violencias de intencionalidad ignorada son en los grupos de edad más avanzada en Brasil (en mayores de 50 años y particularmente en mayores de 60 años), mientras que se presentan en edades más jóvenes en Argentina (entre 20 y 39 años especialmente).

\section{Discusión}

Los sistemas de información de mortalidad producen información sobre la tendencia temporal de las muertes en general y de muertes por violencias en particular. Conocer la magnitud y características de las muertes por violencias depende de la calidad de los sistemas de información de mortalidad, y en particular de la dimensión de las muertes por violencias de intencionalidad ignorada, analizada en este artículo.

El análisis descriptivo de la tendencia temporal de la calidad de la información estadística de mortalidad de Argentina y Brasil utilizó un periodo reciente extenso (21 años) y un volumen de datos relevante.

Los resultados de esta investigación muestran la mejoría de la calidad de las estadísticas de mortalidad, más marcada en Brasil que partió de una situación más desfavorable. En cuanto al esclarecimiento de la causa básica de defunción en las muertes totales la información mejoró en Brasil, y se mantuvo estable en Argentina; los dos países finalizan el periodo en un nivel semejante. En cuánto a las variables básicas de sexo y edad hubo una mejora significativa en su llenado en los dos países durante el periodo.

El flujo de la información del sistema de información de mortalidad por causas externas es diferente al correspondiente a las otras muertes (no debidas a violencias). Las primeras tienen un recorrido dónde interviene la medicina forense a fin de buscar la causa jurídica de la defunción, además del sector salud que busca la causa básica de defunción; mientras que para las demás defunciones (no debidas a violencias) solo interviene el sector salud.

Esta investigación muestra también una tendencia temporal a la mejoría de la calidad de los registros estadísticos de mortalidad cuando analizamos en particular las muertes por violencias, con una disminución de los registros de muertes por violencias de intencionalidad ignorada.

Se observó un leve incremento del porcentaje de muertes por violencias de intencionalidad ignorada en mujeres hasta el 2005 que luego desciende al final del periodo (2010). En ese sentido es necesario investigar sobre las posibles causas del aumento de las muertes de intencionalidad ignorada en mujeres hasta 2005, que podrían corresponder a hechos de violencia doméstica o violencia de género. Por otro lado la magnitud de 
las muertes por armas de fuego de intencionalidad ignorada sigue siendo importante, a pesar de una tendencia temporal a la disminución.

Varios factores contribuyen para el no esclarecimiento de la causa básica de defunción, cuando se trata de una muerte por violencias, como los que son listados a continuación: precario funcionamiento de los Institutos de Medicina Legal o Morgue Judicial; inexistencia de estas instituciones en algunas jurisdicciones; médicos legistas que informan solo la lesión sin mención de la circunstancia que provocó el óbito (que permitiría identificar la intencionalidad); deficiencia en la formación médica y técnico-administrativa para el correcto llenado de los registros de óbito; poca consciencia sobre la importancia de la información y del llenado correcto de los registros estadísticos; supuesto compromiso para el médico legista cuando asigna una circunstancia que podría potencialmente relacionarse con la causa jurídica del óbito (aunque se encuentre protegido por el secreto estadístico); omisiones de hospitales y comisarias que no completan correctamente los instrumentos correspondientes, entre otros factores ${ }^{23-26}$.

Muchas veces la calidad de la información depende de la búsqueda activa de un funcionario que intenta identificar la causa de óbito y las variables de interés para el llenado adecuado de la declaración de óbito, como es recomendado por la Clasificación Internacional de Enfermedades ${ }^{10}$.

Un aspecto no abordado en esta investigación es la calidad de la certificación de la causa básica de defunción cuando está bien definida. Observamos que los responsables de elaborar los registros de mortalidad tienden a colocar en mayor medida una causa básica de defunción específica (definida), aunque no podemos conjeturar si la causa colocada es correcta a partir de esta investigación.

Otra cuestión a tener en cuenta es que se tratan de resultados a nivel nacional y que existe todavía una gran variabilidad en cuanto a la calidad de la información sobre mortalidad en el interior de cada país, en jurisdicciones (provincias y estados) y municipios ${ }^{27-30}$, y por este motivo es necesario conocer con mayor precisión cuál es la cobertura del sistema de información de mortalidad.

Se ha descripto la mejoría de la tendencia temporal de la calidad de la información en muertes por violencias en subunidades nacionales ${ }^{31}$, y se han propuesto diferentes metodologías para su mejoramiento $\mathrm{o}^{32-33}$ o estimación de las omisiones $^{34}$, así como la necesidad de su complementación con otros sistemas de información ${ }^{35}$.

La deficiente calidad de los sistemas de información de mortalidad está relacionada con una concepción de los sistemas de información en salud como sistemas normativos, que no tienen en cuenta los diferentes actores que lo conforman y que les imprimen sus lógicas e intereses ${ }^{36}$. En el caso que nos compete, y a modo de ejemplo, podemos mencionar la relevancia del médico legista en la clasificación de la intencionalidad del tipo de muerte ya que no se trata del médico que pertenece al sistema de salud, sino a la justicia o seguridad pública según el país. Es decir, la concurrencia de múltiples actores con sus lógicas particulares, a veces contrapuestas, dificultan que la información sobre muertes por violencias sea de adecuada calidad para la definición e implementación de políticas públicas.

En este sentido la construcción de la información sobre mortalidad por violencias compromete varios sectores -salud, policía, justiciaque trabajan con distintas dinámicas, flujos y conceptos (por ejemplo: causa básica de defunción para el sector salud y causa jurídica para policía y justicia; o muerte por accidente de tránsito para salud y homicidio culposo para policía y justicia $)^{37}$.

La mirada de esta investigación sobre la calidad de los sistemas de información de mortalidad tiene sus limitaciones ya señaladas anteriormente y debe complementarse con otros tipos de metodologías dentro del sector salud, y también con las otras miradas de sectores que estudian el problema de las violencias (policía, justicia). El análisis de la calidad de los sistemas de información de mortalidad por violencias permite detectar problemas y orientar acciones para obtener información de mayor calidad a fin de orientar políticas públicas preventivas. 


\section{Colaboradores}

M Alazraqui, H Spinelli, MG Zunino y ER Souza trabajaron en la concepción del artículo, en el análisis de los datos presentados y en la redacción de la versión final.

\section{Agradecimientos}

Los autores agradecen a Carlos Guevel y Adrián Santoro de la Dirección de Estadísticas e Información de Salud del Ministerio de Salud de Argentina por organización de los datos estadísticos de mortalidad de Argentina; y a Bruna Soares Chaves Correia por la organización de los datos estadísticos de mortalidad de Brasil.

\section{Referencias}

1. Costa JSD, Victora CG. O que é "um problema de saúde pública”? Rev Bras Epidemiol 2006; 9(1):144146

2. Souza ER, Minayo MCS. O impacto da violência social na saúde pública do Brasil: década de 80 . In: Minayo MCS , organizadora. Os muitos Brasis: saúde e população na década de 80 . Rio de Janeiro: Hucitec; 1995.

3. Alleyne GAO. La prevención de la violencia: compromiso para la Organización. Rev Panam Salud Pública 1999; 5(4/5):i-ii.

4. Krug EG, Dahlberg LL, Mercy JA, Zwi AB, Lozano $\mathrm{R}$, editores. Relatório mundial sobre violência e saúde. Genebra: Organização Mundial da Saúde; 2002.

5. Minayo MCS. A difícil e lenta entrada da violência na agenda do setor saúde. Cad Saude Publica 2004; 20(3):646-647.

6. Minayo MCS. Relaciones entre procesos sociales, violencia y calidad de vida. Salud Colectiva 2005; 1(1):69-78.

7. Franco S. La violencia homicida y su impacto sobre la salud en América Latina. En: Minayo MCS, Coimbra Júnior CEA, organizadores. Críticas e Atuantes: Ciências Sociais e Humanas em Saúde na América Latina. Río de Janeiro: Editora Fiocruz; 2005.

8. Souza ER, Lima MLC, Veiga JPC. Violência interpessoal: homicídios e agressões. In: Brasil. Ministério da Saúde (MS). Secretaria de Vigilância em Saúde. Impacto da violência na saúde dos brasileiros. Brasília: MS; 2005. p.171-203.

9. Organización Panamericana de la Salud (OPAS). Clasificación Internacional de Enfermedades. Washington DC: OPAS; 1978. (Publicación Científica, N.353, V. I y II).

10. Organización Mundial de la Salud (OMS). Clasificación Estadística Internacional de Enfermedades y Problemas relacionados con la Salud, Décima Revisión. Washington DC: OMS; 1995. (Publicación Científica, N.554).

11. Spinelli H. Razón, Salud y Violencia o la (im)potencia de la racionalidad Médico-Científica [tesis]. São Paulo: Universidade Estadual de Campinas (UNICAMP); 1998.

12. Spinelli H, Alazraqui M, Macías G, Zunino MG Nadalich JC. Muertes violentas en la Ciudad Autónoma de Buenos Aires. Una mirada desde el Sector Salud. Buenos Aires: OPS,OMS; 2005. (Publicación Científica, n.60)

13. Zunino MG, Spinelli H, Alazraqui M. Muertes por Armas de Fuego: un eclipse en los sistemas de información en salud. Salud Colectiva 2006; 2(3):259-267.

14. Peres MFT. Violência por armas de fogo no Brasil. Relatório Nacional. San Pablo: Núcleo de Estudos da Violência, Universidade de São Paulo; 2004.

15. Peres MFT. Mortalidade por armas de fogo no Brasil 1991-2000. Brasilia: Ministério da Saúde, OPAS, OMS; 2005 
16. Peres MFT, Santos PC. Mortalidade por homicídios no Brasil na década de 90: o papel das armas de fogo. Rev Saude Publica 2005; 39(1):58-66.

17. Zunino MG, Souza ER, Lauritzen B. Estudio epidemiológico comparativo de la mortalidad por armas de fuego en Brasil y Argentina, 1990-2005. Salud Colectiva 2008; 4(3):349-361.

18. Souza ER. Masculinidade e violência no Brasil: contribuições para a reflexão no campo da saúde. Cien Saude Colet 2005; 10(1):59-70.

19. Couto MT, Schraiber LB. Homens, saúde e violência: novas questões de gênero no campo da saúde coletiva. In: Minayo MCS, Coimbra Júnior CEA, organizadores. Críticas e atuantes: ciências sociais e humanas em saúde na América Latina. Rio de Janeiro: Fiocruz; 2005. p. 687-706.

20. Gomes R. A dimensão simbólica da violência de gênero: uma discussão introdutória. Athenea Digital 2008; 14:237-253.

21. Statistics in Canada. Health Statistics Division. Comparability of ICD-10 and ICD-9 for Mortality. Ottawa: Health Statistics Division; 2005.

22. Anderson RN, Miniño AM, Hoyert DL, Rosenberg HM. Comparability of cause of death between ICD9 and ICD-10: preliminary estimates. National Vital Statistics Report 2001; 49(2):1-32

23. Njaine K, Souza ER, Minayo MCS, Assis SG. A produção da (des)informação sobre violência: análise de uma prática discriminatória. Cad Saude Publica 1997; 3(3):405-414.

24. Jorge MHPM, Gawryszewski VP, Latorre MRDO. Análise dos dados de mortalidade Rev Saude Publica 1997; 31(Supl. 4):5-25.

25. Drumond M, Lira MMTA, Freitas MD, Nitrini TMV, Shibao K. Avaliação das informações de mortalidade por acidentes não especificados e eventos com intenção indeterminada. Rev Saude Publica 1999; 33(3):273-280

26. Njaine K, Reis AC. Qualidade da informação sobre acidentes e violências. In: Brasil. Ministério da Saúde (MS). Secretaria de Vigilância em Saúde. Impacto da violência na saúde dos brasileiros. Brasília: MS; 2005. p. 314-340.

27. Reichenheim ME, Souza ER, Moraes CL, Jorge MHPM, Silva CMFP, Minayo MCS. Violência e lesões no Brasil: efeitos, avanços alcançados e desafíos futuros. The Lancet. Saúde no Brasil 2003; 75-89.

28. Spinelli H, Alazraqui M, Zunino G, Olaeta H, Poggiese $\mathrm{H}$, Concaro $\mathrm{C}$, Porterie S. Firearm-related deaths and crime in the Autonomous City of Buenos Aires, 2002. Cien Saude Colet 2006; 11(2):327-338

29. Waiselfisz JJ. Mapa da Violência 2010: anatomia dos homicídios no Brasil. São Paulo: Instituto Sangari; 2010.

30. Waiselfisz JJ. Mapa da Violência dos Municípios Brasileiros 2008. Brasília: Ministério da Sáude; 2008.
31. Lozada EMK, Mathias TAF, Andrade SM, Aidar T. Informações sobre mortalidade por causas externas e eventos de intenção indeterminada, Paraná, Brasil, 1979 a 2005. Cad Saude Publica 2009; 25(1):223-228.

32. Campos D, França E, Loschi RH, Souza MFM. Uso da autópsia verbal na investigação de óbitos com causa mal definida em Minas Gerais, Brasil. Cad Saude Publica 2010; 26(6):1221-1233.

33. Jorge MHPM, Gotlieb SLD, Laurenti R. O sistema de informações sobre mortalidade: problemas e propostas para o seu enfrentamento. II - Mortes por causas externas. Rev. Bras. Epidemiol. 2002; 5(2): 212-223.

34. Cavalini LT, Ponce de Leon ACM. Correção de sub-registros de óbitos e proporção de internações por causas mal definidas. Rev Saude Publica 2007; 41(1):85-93.

35. Coeli CM, Barbosa FS, Brito AS, Pinheiro RS, Camargo Júnior KR, Medronho RA, Bloch KV. Estimativas de parâmetros no linkage entre os bancos de mortalidade e de hospitalização, segundo a qualidade do registro da causa básica do óbito. Cad Saude Publica 2011; 27(8):1654-1658.

36. Alazraqui M, Mota E, Spinelli H. Sistemas de Información en Salud: de Sistemas Cerrados a la Ciudadanía Social. Un Desafío en la Reducción de Desigualdades en la Gestión Local. Cad Saude Publica 2006; 22(12):1547-1556.

37. Alazraqui M, Spinelli H, Wilner A, Olaeta H. Análisis Cualitativo de la Calidad de Información de los Sistemas Estadísticos de Salud y de Justicia sobre Muertes por violencias en la Ciudad Autónoma de Buenos Aires en 2001. Buenos Aires: Programa de Vigilancia de la Salud y Control de Enfermedades (VIGI+A), Ministerio de Salud y Ambiente de la Nación; 2004.

Artigo apresentado em 10/05/2012

Aprovado em 23/07/2012

Versão final apresentada em 13/09/2012 